\title{
The Effect of Social Media on Identity Construction
}

\author{
Uğur Gündüz \\ Assoc.Prof.Dr., Istanbul University \\ Faculty of Communication, Istanbul, Turkey
}

Doi: $10.1515 / \mathrm{mjss}-2017-0026$

\begin{abstract}
The social media platforms have a growing importance in our lives since they are the places where we "showcase" our living experiences. They also reflect a variety of dimensions regarding our position in the virtual and physical social life. Both of these factors make people to play certain characters in the social arena. The Social Network is gaining more and more importance in today's world and has a deeper impact on the society as to the traditional media. Social media enables identity expression, exploration, and experimentation; something natural for the human experience. It is the agencies in real life, which provide a source of names for different sectors, that inspire the internet communities and the interactions they make within themselves. It is essential to comprehend the motives of agencies to have an understanding of the group interactions on social platforms. The enable individuals present themselves to others and determine the way they would like to be perceives in addition to helping them connect and interact with people, and participate in the activities they wish. Communicating online offers many ways to connect with others: individuals may or may not use their real names, and they can open as many accounts as they want to. This study explores practical aspects of identity construction, relating to issues virtual communities and social media. It also analyzes the probable reasons that individuals feel the need to create a virtual identity for themselves as well as "the spiral of transformation", that is, the creation period goes ahead of the internet to reach the real life. This study also aims at concentrating on the virtual communities appearing in the social networks while questioning their social and cultural qualities and values.
\end{abstract}

Keywords: Social media, virtual community, identity, construction, social networking

\section{Introduction}

Platforms where all digital/virtual users with internet access can share content, express opinions about a topic and where communication and interaction processes intensely take place without any time or place limitation are defined as social media. Via social media, users come together with other users and are included in the communication or interaction processes. They can help other users, receive help from other users, answer the questions of other users and receive replies to their questions from other users. In this aspect, social media is one of the platforms where communication and interaction are most intensely experienced.

Technological convergence and digitization, which allow traditional media to be carried to new types of media, have allowed audio, data and mass communication to take place in the same channel. Due to the internet making mutual data communication possible at the level of web 2.0, the concept of social media has emerged. With the development of social media, circulation of information and news has taken on a structure that should be considered carefully given that its speed and dimensions are quite novel.

Social media allows its users to establish contact with publicly known or unknown users to share a content, which the user likes, with other users. However, it should be kept in mind that 
other users can see information shared via social media. Technological developments that cause the current age to be named as the information age have also led to improvements in data transfer, information sharing and information acquisition processes. With online platforms, mobile communication instruments, cameras, video talk and so forth, and the fact that both electronic systems and interactive systems meet with users have added a new dimension to communication and interaction.

Online access provides drastically superior opportunities than previous communication technologies, leading to the fact that modern-day communication environments are defined as new media. Contents created by people and sharing of these contents, rapid dissemination and adoption of social media have created a social dimension to modern-day communication platforms

Social communication platforms include communication and interaction that are established among users via online platforms. Due to the fact that social communication platforms enable users to transfer images, videos and information, all users can access all contents which they want to reach. Contents created by professional users enable non-professional users to create and share contents thanks to social communication platforms.

Social communication platforms or social networks allow people to share their photographs and events with friends and to follow profiles and events of their friends by creating a digital identity. Facebook, Linkedln, Myspace, Google Plus and Periscope, the popularities of all of which have been surging, are examples of commonly used social networks. The common characteristics of these social networks are that contents are produced by users and are shared with people who are in the same platform.

\section{Aims and Methodology}

New trends of being a citizen of the 21st century lie in the success of the use of communication concepts such as technology based communication, information literacy, media literacy, information bombardment, effective decision making, etc. Media is not only an entertainment tool but also more likely to be an infotainment tool. For some, it's a way to escape from the reality, to some others, it's the virtual reality; yet for a great sum of people it is sometimes more important than the real reality.

Spending so much time in front of screens and sharing files, life stories, profiles, opinions, etc through a more interactive and systematic way, future people seem to be more active or less healthy with their pseudo-names, fake profiles, multiple identities and diversifying attitudes as well as endless efforts to position themselves regarding each piece of news. Thus, the social media brings us clusters, labels, discriminations and inequalities.

The individual needs others to establish himself/herself. The self or identity has a psychological dimension such as feeling the self as a part of a greater body, sense of security, belonging to a social, emotional, political body or community with affective bonds of solidarity. The self or identity has a psychological dimension such as feeling the self as a part of a greater body, sense of security, belonging to a social, emotional, political body or community with affective bonds of solidarity. Identities also bring diversity: Each new identity has its compatibilities and incompatibilities. Individuals might reflect themselves differently in unexpected conditions and layers of communication. Each new layer of communication seems to require a different identity; each new grouping will blur or clarify, depending upon the others in the communication. Virtual networks create diversity in society, so reflexive society creates reflexive identities. The risk of stereotyping also impacts, and there will be many identities resembling each other in the same community. (Gunduz\&Pembecioglu, 2014, p.39)

The main purpose of this study is to develop a framework for understanding and analyzing social media as an autonomous social structure in which to construct an identity. This study also explores practical aspects of identity construction, relating to issues virtual communities and social media. It also analyzes the probable reasons that individuals feel the need to create a virtual identity for themselves as well as "the spiral of transformation", that is, the creation period goes ahead of the internet to reach the real life. This study also aims at concentrating on the virtual communities appearing in the social networks while questioning their social and cultural qualities and values. 


\section{New Communication Technologies: The Concept of Social Media and Social Networks}

The structural advantages and advanced applications of new communication technologies offer an environment of freedom and ease content customization. There is a stark contrast between the forced consumption style and feeling of restriction presented to the audience by traditional media and the feeling of comfort and freedom presented to the new media audience.

As a result of the interaction between technical infrastructure and communication concepts, information has become easily transmittable to wide communities; currently, people can access needed information without the delay that would be experienced with traditional communication systems (Karasar,2004,p.117).

Modern-day communication systems quantitatively and qualitatively resemble each other. Watching TV on portable devices or online surfing on a fixed TV at home have mostly become routine. However, integration of new communication systems with one another shapes communication processes and enables communication in the light of personal necessity and desires (Karacor,2009,p.121).

The "new" term used in the new communication technologies is preferred because it has undergone a transformation. When the "new" term is evaluated in respect to communication systems, a newly generated system benefits from the previous system. The fact that new communication systems establish communication and interaction in a way that can be alternative to the former communication system is the basis of communication technologies of our age (Vural\&Bat,2010,p.3349). According to Erdem (2012,p.183), "emerging of the new communication technologies was interpreted as a revolution, this revolution also brought about a social revolution. Claims of that all people in the world are linked each other via a virtual channel and freedom of communication, participation, development and democratization claims are also in the world's agenda."

While creating alternative communication systems, previous communication systems should not be ignored; new systems should include attributes that improve both mass and personal communication. In regards to data transfer time, data transfer volume, place and date storage features, channels used in new communication technologies provide ease of use (Calik,2010,p.4).

Media presents a virtual framework for all individuals within a society to live within and brings this framework into the forefront as a settlement location. In other words, it creates an influence area within which an item which does not have an appearance in the media has no chance to make its presence felt (Gunduz,2013,p.134). Social communication platforms are one of today's most important issues due to the fact that users share their opinions, emotions and thoughts without having to practice any censorship or restraint and within which contents are created by users. Therefore, both organizations and non-professional users look for people who have common emotions, thoughts and opinions with themselves by sharing on social communication platforms, becoming an active power by constituting a community with like-minded users.

Social communities are communication platforms where people share online their common tendencies with acquaintances and interact with their acquaintances. Social communities allow people to create a profile and share their own photographs and events with their friends and to follow profiles and events of their friends (Calik, 2010,p.4). "Rapid development experienced in communication technologies brings every culture of the world closer each other. As a result of this, interaction of every culture of the world with other culture has increased" (Karacor,2009,p.121).

It can be said that social networks will become a form of media in the future. It has been estimated that in the face of ease of content selection and production of social media, traditional media cannot stand in the long term, although it should persist in the short and medium term. Although social media has unique rules, it is a fact that its deep-rooted principles have not yet been established. It can be thought that in the next time periods, at least in the medium term, it will undergo fundamental changes. With these changes, there are sufficient reasons to believe that social media, which is fully developed in terms of new income sources and sourcing, will be more powerful against the traditional media and will reshape all communication channels. 


\section{Virtual Socialization and Virtual Communities}

It is possible to regard effective communication as belonging to a group, a nature of remaining a part of a group. In this framework, people fulfill their socialization functions when they perform actions such as confirming known things, liking or disliking posts, and even making comments about them and reacting to other comments. The most preferred action is likely to upload interesting photographs or videos that are not known by others given that new media viewership has rapidly taken the place of TV and other viewership.

The necessity of people to watch one another has switched to what other people are doing on their mobile phones rather than reading signs, advertisements, posters or other people's newspapers. In other words, everyone is under watch now. Consider that you are traveling with an audience, always checking others to see whether they are playing a game, messaging or watching a movie during a bus trip or even while getting on or off the tramway. Moreover, observing what other people are doing causes attitude, opinion and behavior changes. Therefore, the same audience finds social network messages more appealing than traditional media.

Daniel (2011, p.24) claimed that there was an unprecedented rise in the number of internet communities in the twenty-first century, which shows that people strongly wish to socialize, share things, play games and work together regardless of time, space or the distance between them. A number of platforms including Facebook, Twitter, MySpace, Blogs, Wikis and Linkedln have a growing popularity as an assistance for everyday activities. It is the social systems that shape the conventional ways of studying, and the integration of the modern technologies into our lives has demonstrated that some of these ways are insufficient to have an insight about the lives of people with social and cultural dimensions. There is need for scientific methods and perspectives to comprehend internet communities now that the virtual groups and happenings within the technologies became obvious. It is vital to have this understanding to generate beneficial information systems with no weaknesses as well as new tools and effective procedures. It will also help us maintain more meaningful interactions on the internet without any dangers yet with more fun instead.

Virtual environments are digital platforms where people establish communication with each other and give support to produced content on online platforms. In virtual environments, people are included in a common area whenever they want. People create a profile that represents themselves and are in interaction with others aurally or in written form. Thanks to virtual environments, people can form groups of all sizes (Islek,2012,p.34). Although virtual environments are specified as virtual, the environment where they take place and the communication in that environment are realtime. Social interaction is becoming very easy thanks to virtual environments.

Use of virtual environments in both the commercial and educational fields has rapidly become widespread. Currently, educational applications have become popular, partially via distance education used by universities. Free from pressures originating from the super-ego, users can express themselves freely in virtual platforms. Users getting together via virtual platforms escape from their roles and share freely.

Virtual communities whose existence is primarily online, as opposed to physical world communities who conduct some but not all of their activities offline, are at a disadvantage in that there is little in the network or in network technology as currently constituted that encourages commitment. There are few "no exit" relationships in cyberspace, and the term "surfing the net" is an apt descriptor of the depth of much online activity (McLaughlin,Osborne\&Ellison,2011,p.149). In communication processes, internet use allows people to establish interaction by ignoring voice, image and biological and social gender for the first time. Users can establish an identity for themselves by creating any profile that they want and can freely share any information (Boyd \& Ellison,2012,p.1).

In virtual environments, a user can establish an interaction with another user that they do not know without using his/her identity or by hiding or changing his/her gender or social status. Communication processes in virtual environments are carried out using fictionalized profiles (Otrar\&Argin,2014,p.2).

Virtual environments differentiate from traditional interactions by the fact that users can 
express themselves freely, separate from pressures originating from the super-ego. Creations in virtual environments have also some traditional aspects however. Every environment that develops the feeling of community is affected at least a little by real life roles (Akyazi\&Unal,2013,p.4). Castells (2005,p.479) when asking whether virtual communities are real, gave the answer "They are, but not physical; they do not follow interaction and communication patterns followed by physical communities".

When users who defend a political view in real life get together with people defending the same view, it is not possible to include communication processes ignoring values of that view. In other words, although the expression is virtual, it is also affected by real life.

\section{An Overview of Social Media and Virtual Identity Concept}

Digital identity is attitude norms that are blended with technology. Digital identity includes displaying ethical and appropriate attitude while using electronic environments and receiving information about using electronic environments (Ozkan,2013,p.387).

Digital identity should make an impression that can criticize, has an idea about ethical results of initiatives conducted in interactive environments, can establish an ethical interaction, and does not abuse informatics, virtual environment and social media while using electronic interaction tools and environments. It is expected that a user with a digital identity act ethically and collaboratively when establishing interactions in virtual platforms (Ozel\&Sert,2014,p.303). To clarify digital identity, it is helpful to present its dimensions.

Digital trade and communication dimensions aim to provide competence and technological infrastructure for performing commercial activities in virtual environments. Digital communication has added a new dimension to communication processes and highlights the importance of interactive environments. Digital communication methods such as electronic mail, mobile communication instruments, and instant messaging systems have added a new dimension to personal and community interaction.

In today's technological world, digital literacy has gained importance, just as media literacy in previous ages. Accordingly, technology use involved in activities such as researching a topic, learning and teaching has become an obligation, not a choice. Both people and commercial and non-commercial organizations have become aware of the importance of technology use in the learning and teaching processes.

"Like many other communication platforms, avatars also constituted a different style literacy requirement for digital environments. As does the intensity of digital life in social experience increase, gaining this literacy has been seen more important day by day. In digital multiuser environments, to establish and maintain communication with other users, it will be beneficial to be aware of the fact that an avatar of one user can convey which information and his/her own avatar can imply what things to other users" (Sengun,2014,p.36).

To mention the concept of digital ethics; users should not ignore the fact that an attitude presented or a transaction executed in the internet environment also have an electronic standard. Although internet and social media are virtual users' attitudes and transactions should not have the intention to harm the rights and interests of others. Harming of rights and interests of others in the virtual environment is commonly manifested as bullying or defamation and similar cases.

Legal arrangements are an irrevocable part of social life. Digital law includes liability of transactions executed in the internet environment in terms of digital identity. These transactions are subjected to particular punishments according to laws and regulations. The liability of transactions executed in the internet environment in terms of digital identity means that making banned broadcasts, promoting or selling a product of which sale is prohibited by law, thematic broadcasts encouraging to suicide and activities are subject to penal sanction.

Digital rights and liabilities can be evaluated in the following context: Although there are some limitations on the internet environment, the basis of this is a dynamic built on the fact that people express themselves freely. In the internet environment, people have rights and liabilities for expressing themselves freely. These rights and liabilities include some processes such as expressing an opinion on social media, forums or groups, or holding a discussion about a topic. 
When the answer of the question 'What is digital health?' is being sought, it is possible to find the following result: People should be aware that there can be some factors in the internet environment that physically, mentally or spiritually affect health directly or indirectly. These effects include visual health, stress, introversion, and physical problems.

In the face of present-day technological developments, the importance of the security concept has appeared. Taking measures that protect user's personal security in the internet environment are evaluated within the scope of digital security dimension. Users should not share their password information with others on blogs, microblogs and similar platforms where users register on social media and virtual environments. Additionally, users should not open e-mails that come from unknown sources. It is quite easy to steal personal information via viruses uploaded to e-mails that come from unreliable sources. The first measure to be taken is to not share password information with others. The second important measure is to use a licensed anti-virus service (Ozel, 2011,p.12).

When it comes to creation and acquisition of digital identity, digital identities are certifications of users in one respect. They confirm and allow the recipient to certify that an e-mail was really sent by you. Like identity cards used in daily life, a digital identity is also used to introduce oneself to others. Digital identity prevents another user from taking one's place and acting as this person, establishing communication and interaction on behalf of this person (Islek,2012,p.32).

Standard e-mails that are sent via e-mail addresses established to build lines of communication in the virtual environment are digital versions of postcards used in real life; they can be opened and changed in the transfer process. It is better to encrypt digital identities, hide the content of the e-mail and ensure that no changes can occur during the transfer process via mail message applications. In many organizations, members are assigned a digital identity by an information system technician. For personal use, it is necessary to get a digital identity from a certificate manager (Ozkan, 2013,p.388).

In today's social media world, concepts such as digital tagging and social bookmarking have gained importance. Tagging is used for both real and digital applications. Digital tagging provides information about the content and gives connection to a particular user, a web site or a place regarding visual or other types of media sharing. Digital tagging is used to determine which users are included in an image shared or to inform about the image or content shared (Balaman\&Karatas,2012, p.498).

It is possible to broaden the scope of the message by including people, opinions, thoughts and tendencies using digital tagging. Facebook and Twitter users can directly involve a person in an issue by tagging content that they share. For example, a popular Twitter user can tag a mayor on his/her posts and that major thus can directly be an object of the content. In this way, subject follow-up can be widely performed and users with similar emotions and thoughts can be involved in the process. In short, it is possible to state that social media is a communication stage where people opposing the system and people dominating the system come together. Therefore, "social media belongs to a cyber-world that real and virtual, real and story, real and lie have intertwined each other" (Erdem,2012,p.167)

Social media is regarded as having unique language and oratory culture. It has been observed that oratory tradition, which is a holdover from days when oratory arts were dominant and writing was not yet widely used, still influences written culture. "Oratory is an art of public speaking, delivering a speech in front of an audience. It has two aims including to persuade and to explain" (Ong,2012,p.131). Politicians, artists, and opinion leaders can write long posts by splitting them into numbered parts. Tweets can be shared with determining numbers (1-2-3...) and can reach the masses. This can be regarded as a continuation of the oratory tradition.

Social media allows its users to establish contact with publicly known or unknown other users, to share favored content with other users. However, it should be kept in mind that other users can see information shared via social media. Nowadays, social media being in the public eye has become an indispensable fact for everyone, famous or not.

\section{Conclusion}

Social media has become a widely discussed media form, which nonetheless is in plain sight, due 
to the fact that users can share their opinions, emotions and thoughts without having to impose selfcensorship and because its content is created primarily by users. It is in question whether social media, which has gained strength from its effect on the masses, reaches different segments of society. Both organizational structures and non-professional users look for people who have common emotions, thoughts and opinions with themselves by sharing on social communication platforms and they turn into an active power by constituting a community with like-minded users.

Although virtual media are specified as virtual, the environment where they take place and the communication with other people in that environment are in real-time. Social interaction is becoming easier thanks to virtual environments, a situation that allows people to socialize in virtual environments. Separate from pressures originating from the super-ego, users can express themselves freely in virtual environments. Users getting together via virtual environments escape from their roles and can share freely.

Opinion, thought and tendencies shared about a message using digital tagging are likely to broaden the scope of the message in a way that can be seen by other users. Facebook and Twitter users can directly involve a person in the issue by tagging content that they share. Such rapid access and admission of social media into our lives is closely associated with the change experienced in the social sphere. The structure of social communication platforms has the ability to display the social structure of the current age. Social movements have flexible organization qualities as do economic issues, and it has been seen that social media is an instrument that enables flexible organization. In the name of social movements, the internet has fully appeared as an alternative media. Alternative media has risen to prominence while the mainstream media remains inadequate and ineffective due to various reasons. Social media and networks developed by people not only constitute the parts of network society but also substitute the organized society ideal, which is not achieved at the basis of civil society through social media.

Periods when the media has transformed as a goal rather than a tool have been often experienced in Turkey as in many countries. Audiences of both traditional media and social media want to make themselves visible. Media is aware of every issue, is a remedy for every trouble and makes non-visible things visible. Mass media is also formed by data for the sake of mass itself. Given such a wide and heterogeneous audience with a public structure but without an identity, actualization speed of events and circumstances require complex formal institutions.

As a result, social media is a magic wand that determines structure of the society, forms a basis for polarizations and dissolutions and also ensures mergers and agreements. The Big Brother concept in George Orwell's novel Nineteen Eighty-Four is now valid for everyone and has caused a panopticon in that everyone observes everyone. In this platform, each person can replicate hundreds of times and will continue to replicate in the expectation that this replication will provide a kind of happiness while their reflections move away from their realities.

On one hand social media allows people to express themselves, on the other hand it takes their believability and reliabilities away, trivializes them by objectifying. "Mainstream media" can be formed within the frame of social media in one sense. Although this situation appears as if it has emerged by itself, attractiveness of the like button, a profile of friend/follower list that forces participation lead people to become such that he/she has to plan all actions to be performed with a feeling of being kept under close watch continually.

\section{Acknowledgement}

Istanbul University's SRPCU (Scientific Research Projects Coordination Unit) provided assistance to the conduct of this study (Project ID: BEK-2016-21217).

\section{References}

Akyazi, E. \& Unal, A.T. (2013): "Purpose, Adoption and Level of Loneliness Relation and the Use of Social Networks: A Study on Undergraduate Communication Students", Global Media Journal, 6(3), 1-24.

Balaman, F. \& Karatas, A. (2012): "The Usage Purpose of Social Network Sites of High School Students and Social Network Elements", Batman University Journal of Life Sciences, 1(1), 497-504. 
Castells, M. (2005): The Rise of the Network Society, With a New Preface: Volume I: The Information Age: Economy, Society, and Culture, Istanbul: Bilgi University Publications.

Calik, D. (2012): "New Communication Technologies and the Reflections on the Organizational Communication", Paper presented at 17. The Conference Internet in Turkey, 7-9 November 2012 Eskisehir Anadolu University, Retrieved at August 5, 2016 from http://inet-tr.org.tr/inetconf17/bildiri/17.pdf, $1-9$.

Boyd, D. \& Ellison, N.(2008): "Social Network Sites: Definition, History, and Scholarship", Journal of Computer Mediated Communication, 13(1), 210-230.

Daniel, Ben Kei (2011): Handbook of Research on Methods and Techniques for Studying Virtual Communities: Paradigms and Phenomena Vol.1, USA: Information Science Reference.

Erdem, B.K.(2012): "Rhizome of the New World Order: The Arabs and Social Media", All of Us Global, All of Us Local from Gutenberg Galaxy to Zuckerberg Galaxy, ed. by Edibe Sözen, Istanbul: Alfa Publications, 165187.

Gunduz, U.(2013): "Social Movements, Civil Disobedience and The Reflections of Social Media", Büyükaslan, A. \& Kırık A. M. (ed), Researches on Social Media-1: Socializing Person, Konya: Cizgi Publishing, 133154.

Gunduz, U. \& Pembecioglu, N.(2014): "The Role of Social Media in the Sharing and Delivery of Authoritative and Governmental Issues", European Journal of Research on Education, Special Issue: Contemporary Studies in Social Science, ISSN: 2147-6284, 24-40.

Karacor, S. (2009): "New Communication Technologies, Political Participation, Democracy", Manisa: Celal Bayar University The Journal of Management and Economy, 16(2), 121-131.

Karasar, Ş.(2004): "New Communication Technologies in Education: Internet and Virtual Higher Education", The Turkish Online Journal of Educational Technology, 3(4), 117-125.

McLaughlin M. L, Osborne K. K. \& Ellison N. B. (2011): "Virtual Community in a Telepresence Environment", Virtual Culture Identity and Communication in Cybersociety, ed. by Steven G. Jones, U.K: Sage Publications, 146-168.

Ong, J. W.(2012): Orality and Literacy, Technologizing of the World, trans. by Sema Postacioglu Banon, Istanbul: Metis Publishing.

Otrar, M. \& Argın, F. S. (2014): "The Examination of the Students' Attitudes Towards Social Media within the Context of Habits" Journal of Research in Education and Teaching, 3(3), 1-13.

Ozel, A. P.(2011): "Social Media and Trust: An Empirical Research Directed to Government, Non-Governmental Organizations and Business, Academic Journal of Information Technology, 7(2), 1-31.

Ozel, A. P. \& Sert, N.Y.(2014): “Corporate Blogs as a Tool of Digital Public Relations: A Research on Turkey's First 100 Companies",Global Media Journal TR Edition, 5 (9), 303-321.

Ozkan, O. (2004): "The Use of the New Communication Technologies in Political Life and Turkey Example", (Unpublished master thesis) Isparta: Suleyman Demirel University.

Ozkan, N. P.(2013): "The Identity Construction Process of Social Networking Users in E-Socializing Processing", Paper presented at the II. International Conference on Communication, Media, Technology and Design, 2-4 May 2013, Famagusta North Cyprus, 386-391.

Sengun, S.(2014): "A Semiotic Analysis for Digital Avatars and The Roles of Reducing Uncertainty in Digital Communication", Paper presented at the International Academic Conference of The Impact of Digital Communication Istanbul: Iskenderiye Publishing, 33-44.

Vural, B. A. \& Bat, M. (2010): "Social Media As a New Communication Environment: A Research on Ege University Faculty of Communication", Journal of Yasar University, 20(5), 3348-3382. 